\title{
ANÁLISE NUMÉRICA DE VIBRAÇÕES EM UMA PASSARELA MISTA DE AÇO E CONCRETO
}

\author{
Numerical Analysis of Vibrations in a Mixed Steel and Concrete Footbridge
}

\author{
Launelle Cardozo Martins ${ }^{1}$, José Francisco Anunciação Pinto ${ }^{2}$, Thiago Bonjardim Porto
}

Recebido em 11 de janeiro de 2017; recebido para revisão em 21 de março de 2017; aceito em 29 de abril de 2017;

disponivel on-line em 16 de maio de 2017.

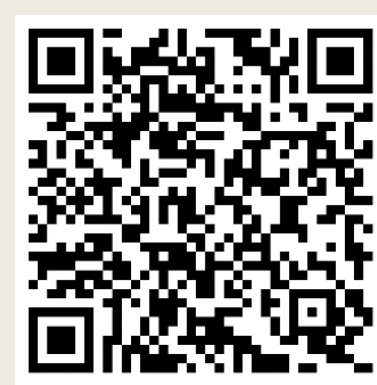

PALAVRAS CHAVE:

Vibrações;

Estruturas mistas;

Análise numérica;

Analise modal;

Modelagem

computacional.

\section{KEYWORDS:}

Vibration;

Composite structures;

Numerical analysis;

Modal analysis;

Computational modeling.

* Contato com os autores:

${ }^{1}$ e-mail: launelle@hotmail.com ( L. C. Martins )

Engenheira Civil, Especialista em Engenharia Civil, Professora colaboradora do Curso de Engenharia Civil da Faculdade

Presidente Antônio Carlos de Teófilo Otoni.

2 e-mail: jose.anunciação@outloock.com ( J.F.A Pinto )

Engenheiro Civil, Mestre em Engenharia Civil, Professor colaborador do Curso de Engenharia Civil da Faculdade de Ciências

Gerenciais de Manhuaçu (FACIG).

3 e-mail thiago@consmara.com.br (T. B. Porto )

Engenheiro Civil, Doutor, Professor colaborador do curso de Engenharia Civil da Universidade Federal de Ouro Preto. 


\section{INTRODUÇÃO}

Atualmente há uma demanda crescente por métodos construtivos mais industrializados, que unam rapidez e eficiência. Além disso, as recentes tendências em design exigem vãos cada vez maiores e estruturas cada vez mais arrojadas, e consequentemente, estruturas mais delgadas. Como resultado, as edificações construídas recentemente muitas vezes não apresentam desempenho satisfatório quanto ao amortecimento de vibrações, principalmente quando comparadas que as edificações mais antigas. Dessa forma, as vibrações provenientes de fatores externos podem se tornar uma fonte de ansiedade e desconforto para os ocupantes.

Segundo Mangerig e Zapfe (2007), as propriedades dos materiais modernos, como o aço, permitem estruturas cada vez mais esbeltas, resultando em menor rigidez, como também no menor peso de construção. Assim, ocorre um deslocamento das frequências características das estruturas para níveis que aumentam o perigo de excitação de ressonância. Além disso, a esbeltez das edificações modernas reduz a inércia resultando em quantidades de energia menores necessárias para iniciar a vibração perceptível. Esses fatores ressaltam a necessidade de uma análise mais minuciosa quanto aos efeitos de vibração e aos Estados Limites de Serviço.

Estruturas Metálicas têm

tradicionalmente uma frequência natural menor, quando comparada as de Concreto Armado. Como consequência direta disso, elas são mais sensíveis a diversos tipos de carregamentos dinâmicos, já que sua frequência natural é próxima a de atividades humanas rítmicas comuns, como o caminhar humano, vento, tráfego de carros, etc. Desse modo, é possível que haja ocorrência de ressonância produzindo elevados níveis de vibração, os quais são capazes de prejudicar o conforto dos ocupantes ou até mesmo comprometer a segurança estrutural.

Ressonância ocorre, quando a frequência de uma onda coincide com a frequência natural de um corpo. Ocorre assim um acréscimo de energia por meio de ondas periódicas, fazendo com que este passe a oscilar com amplitudes cada vez maiores. Existem inúmeros exemplos dos efeitos desastrosos causados por este fenômeno, dentre os quais podemos citar Millenium Bridge, em Londres. No dia da inauguração desta ponte, o caminhar das pessoas sobre a mesma fez com que a estrutura entrasse em ressonância, necessitando, por conseguinte, ser reforçada (DALLARD et al., 2001). Outro caso famoso é o da ponte de Takoma, nos EUA, em que a ponte entrou em ressonância com o vento, o que provocou violentas ondulações, ocasionando a queda da mesma (FULLER et al., 1994).

Levando em consideração o histórico de acidentes e o atual crescimento da influência da atividade humana no comportamento dinâmico das estruturas, fica evidente a escassez de estudos na área de vibração em estruturas metálicas e também a pequena importância que é dada ao assunto durante a etapa de projeto. A NBR 8800 (ABNT 2008) em si apresenta considerações bastante simples a respeito deste fenômeno. De acordo com Ahmed e Badaruzzaman (2011), embora estas vibrações de piso não constituam uma ameaça à integridade estrutural do sistema, ela pode ser tão desconfortável para os ocupantes da edificação que o sistema de piso pode acabar sem utilidade. Portanto, para evitar um problema relacionado à vibração em estruturas é desejável entender seu comportamento dinâmico e considerá-lo na concepção.

\section{OBJETIVO}

O objetivo geral deste trabalho é a realização de uma análise numérica de vibrações em uma passarela de aço e concreto. Além disso, pretende-se simular o caminhar humano nesta estrutura para avaliar os níveis de vibração do sistema estrutural. Ao final do estudo, objetivamos comparar a aceleração obtida com a resposta dinâmica do modelo, com normas internacionais que estabelecem critérios de aceitabilidade em relação ao conforto humano. Desta forma, conseguiremos definir ao final deste artigo se a passarela em estudo está dentro dos padrões 
exigidos de conforto humano, ou não. Dessa forma, este estudo visa ponderar a influência da vibração em estruturas metálicas e sua consequente influência no bem-estar das pessoas. Além disso, objetiva destacar a importância do desenvolvimento de modelos computacionais, pois estes permitem a avaliação da vibração ainda na etapa de projeto. Isso representa um grande avanço para a construção civil, pois permite que a análise estrutural fique mais próxima da realidade, além de possibilitar que sejam feitas alterações, ainda na etapa de projeto, com o intuito de aumentar a rigidez, e consequentemente, a frequência natural. Tenciona também avaliar o comportamento da estrutura quanto à influência no conforto humano, podendo classificá-la de acordo com os limites estabelecidos pelas normas internacionais.

\section{CARACTERIZAÇÃO ANALÍTICA DO} CAMINHAR SEGUNDO O MODELO PROPOSTO POR VARELA E BATISTA

A caminhada é um tipo de excitação extremamente comum em sistemas de pisos. Segundo Mello (2005), durante o ato de caminhar ocorre um movimento organizado de pernas, que causa propulsão para frente, mediante impulsos alternados e rítmicos. Neste processo ocorre um movimento de subida e descida da massa do corpo de aproximadamente $50 \mathrm{~mm}$ em cada passada, conforme mostrado na Figura 1.
De acordo com Mello (2009), a representação matemática da reação do piso pode ser resolvida por uma série de componentes senoidais (série de Fourier). Esta série possui três termos que consideram a parcela estática associada ao peso da pessoa e três ou quatro componentes harmônicos de excitação. Esses harmônicos são gerados devido à interação entre a carga crescente representada por um pé e pelo simultâneo descarregamento do outro pé. Os termos da série de Fourier que compõem a função aproximada podem ser visualizados na Figura 2.

A força dinâmica $P(t)$ que representa o caminhar humano, pode ser representada pela série de Fourier, como indicado na Equação 1 :

$$
\mathrm{P}(\mathrm{t})=\mathrm{P} \times\left[1+\sum \alpha_{\mathrm{i}} \times \cos \left(2 \times \pi \times \mathrm{i} \times \mathrm{f}_{\mathrm{p}}+\varphi_{\mathrm{i}}\right)\right]
$$

\section{Em que:}

$\mathbf{P}(\mathbf{t})=$ representação matemática aproximada da força da reação do piso ao caminhar;

$\mathbf{P}=$ peso de uma pessoa $(\mathrm{N})$;

$\boldsymbol{\alpha i}=$ coeficiente dinâmico do i-enésimo harmônico da frequência da força;

i = número do i-enésimo harmônico da frequência força;

$\mathbf{f p}=$ frequência do passo do caminhar humano;

$\mathbf{t}=$ instante de tempo em segundos (s);

fi = ângulo de fase entre o i-enésimo e o primeiro harmônico.

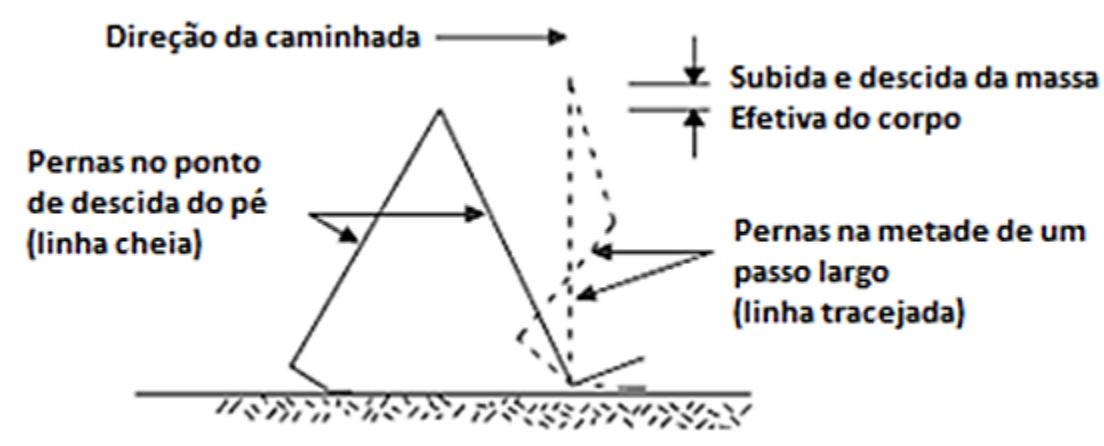

FIGURA 1: Geometria simplificada de uma passada. FONTE: (Figueredo, 2005) 


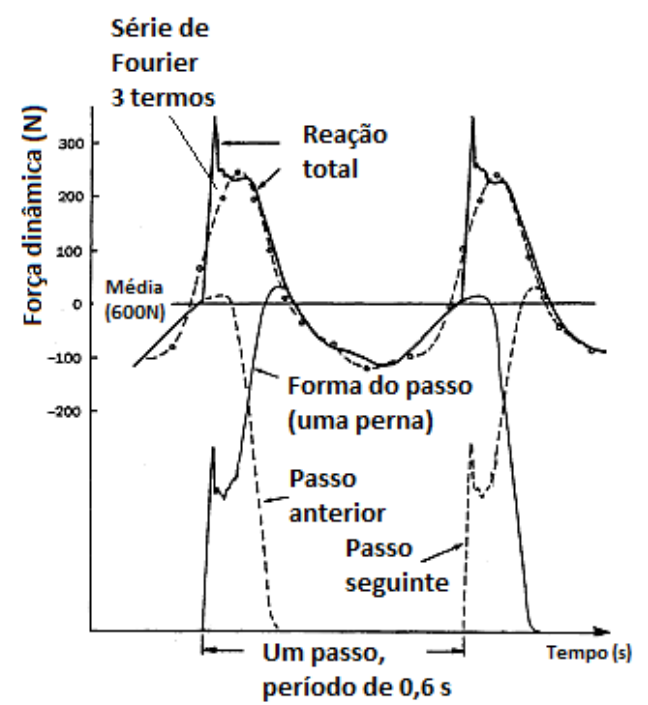

FIGURA 2: Força de contato de um passo e reação do piso.

FONTE: (Varela, 2004).

Em relação ao tamanho de uma laje, a distância entre os pés em um passo é muito pequena, pode-se, portanto, considerar que a força de reação do piso é a soma das forças aplicadas pelos dois pés no intervalo de um passo.
Varela (2004), usando como base a aproximação matemática da reação do piso, propôs uma equação matemática que pudesse representar a magnitude dessa reação. Esta equação foi determinada segundo cinco trechos, que podem ser vistos na Figura 3, e representa matematicamente, trecho a trecho, segundo a Equação 2:

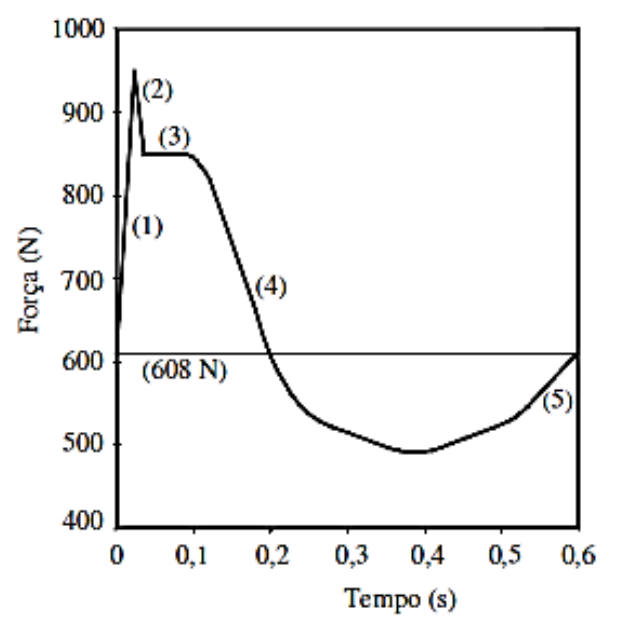

FIGURA 3: Função de carregamento proposta por Wendell.

FONTE: (Varela, 2004).

$$
P(t)=\left\{\begin{array}{ccc}
\left(\frac{\mathrm{f}_{\mathrm{mi}} \cdot \mathrm{F}_{\mathrm{m}}-\mathrm{P}}{0,04 \mathrm{~T}_{\mathrm{p}}}\right) \mathrm{t}+\mathrm{p} & \text { se } & 0 \leq \mathrm{t} \leq 0,04 \mathrm{~T}_{\mathrm{p}} \\
\left.\left[\mathrm{f}_{\mathrm{mi}} \cdot \mathrm{F}_{\mathrm{m}}\left[\frac{\mathrm{C}_{1}\left(\mathrm{t} \cdot 0,04 \cdot \mathrm{T}_{\mathrm{p}}\right)}{0,02 \mathrm{~T}_{\mathrm{p}}}\right]+1\right]\right] & \text { se } & 0,04 \mathrm{~T}_{\mathrm{p}} \leq \mathrm{t} \leq 0,06 \mathrm{~T}_{\mathrm{p}} \\
\mathrm{F}_{\mathrm{m}} & \text { se } & 0,06 T_{p} \leq t \leq 0,15 T_{p} \\
\mathrm{P}+\sum_{\mathrm{i}=1}^{\mathrm{nh}} \mathrm{P} \cdot \alpha \cdot \alpha \cdot \mathrm{s}\left[2 \pi \pi \mathrm{i}_{\mathrm{p}}\left(\mathrm{t}+0,1 \mathrm{~T}_{\mathrm{p}}\right)+\varphi_{1}\right] & \text { se } & 0,15 \mathrm{~T}_{\mathrm{p}} \leq \mathrm{t} \leq 0,90 \mathrm{~T}_{\mathrm{p}} \\
10\left(\mathrm{P}-\mathrm{C}_{2}\right)\left(\frac{\mathrm{t}}{\mathrm{T}_{\mathrm{p}}}-1\right)+\mathrm{P} & \text { se } & 0,90 \mathrm{~T}_{\mathrm{p}} \leq \mathrm{t} \leq \mathrm{T}_{\mathrm{p}}
\end{array}\right.
$$


Em que:

$\mathbf{P}(\mathbf{t})$ : força aproximada da reação do piso ao caminhar;

t: instante de tempo em (s);

P: Peso da pessoa (N)

i: número do i-ésimo harmônico da frequência fundamental da força;

$\boldsymbol{F}_{\boldsymbol{m}}$ : é o valor máximo da série de Fourier e é dado pela Equação 3;

al: Coeficiente dinâmico do i-ésimo harmônico , conforme apresentado na Tabela 1

fp: frequência do passo;

фi: ângulo de fase entre o i-ésimo e o primeiro harmônico;

Pol: amplitude do i-ésimo harmônico da frequência fundamental da força;

$\boldsymbol{f}_{\boldsymbol{m i}}$ : é o fator de majoração do impacto do calcanhar, geralmente tomado como 1,12 ;

$\boldsymbol{C}_{1} \boldsymbol{e} \boldsymbol{C}_{2}$ : são coeficientes dados pelas Equações 4 e 5 ;

nh: números de harmônicos considerados para representar a força;

$$
\begin{gathered}
F_{m}=P\left(1+\sum_{i=1}^{n h} \alpha_{i}\right) \\
C_{1}=\left(\frac{1}{f_{m i}}-1\right)
\end{gathered}
$$

$$
\mathrm{C} 2=\left\{\begin{array}{ccc}
\mathrm{P}\left(1-\alpha_{2}\right) & \text { se } & \mathrm{nh}=3 \\
\mathrm{P}\left(1-\alpha_{2}+\alpha_{4}\right) & \text { se } & \mathrm{nh}=4
\end{array} \quad\right. \text { Eq.[5] }
$$

Destaca-se que os coeficientes dinâmicos associados aos harmônicos da caminhada humana são função da frequência do passo do pedestre. $\mathrm{Na}$ Tabela 1 são mostrados esses coeficientes.

A modelagem computacional do carregamento dinâmico gerado pela ação dinâmica provocada pelo caminhar humano é bastante complexa. O modelo de carregamento utilizado na representação numérica computacional apresenta variação espacial e temporal, visando dessa forma uma representação mais realista do fenômeno do caminhar.

Há fatores como a distância, a frequência e a velocidade do passo do pedestre que influenciam a resposta do caminhar e, portanto, devem ser consideradas na análise. A Tabela 2 apresenta valores destes parâmetros conforme o tipo de caminhada. Dessa forma, para estabelecer o tempo de contato da carga dinâmica com a estrutura é necessário definir de antemão o comprimento e a frequência do passo.

\section{TABELA 1: Harmônicos do passo.}

Harmônico

\begin{tabular}{cccc}
$\mathbf{i}$ & $\mathrm{fp}$ & $\alpha$ & $\phi i$ \\
$\mathbf{1}$ & 1,6 a 2,2 & 0,5 & 0 \\
$\mathbf{2}$ & 3,2 a 4,4 & 0,2 & $\pi / 2$ \\
$\mathbf{3}$ & 4,8 a 6,6 & 0,1 & $\pi / 2$ \\
$\mathbf{4}$ & 6,4 a 8,8 & 0,05 & $\pi / 2$ \\
\hline
\end{tabular}

FONTE: Mello (2005).

TABELA 2: Características do caminhar humano.

\begin{tabular}{cccc} 
Atividade & Velocidade $(\mathrm{m} / \mathrm{s})$ & $\begin{array}{c}\text { Distância do } \\
\text { Passo }(\mathbf{m})\end{array}$ & $\begin{array}{c}\text { Frequência do } \\
\text { Passo (Hz) }\end{array}$ \\
\hline $\begin{array}{c}\text { Caminhada lenta } \\
\text { Caminhada } \\
\text { normal }\end{array}$ & 1,1 & 0,6 & 1,70 \\
Caminhada rápida & 1,5 & 0,75 & 2,00 \\
\hline
\end{tabular}




\subsection{CRITÉRIOS PARA ACEITAÇÃO QUANTO AO CONFORTO HUMANO}

De acordo com Fanella e Mota (2015), muitos critérios foram propostos ao longo dos anos relacionados à vibração e ao conforto humano, no entanto, até a data, não existem padrões universalmente aceites. Logo, a discussão sobre andar e excitação rítmica segue. Segundo o mesmo autor, vibrações podem ser induzidas por uma pessoa ou pessoas andando em um sistema de piso. Dependendo de vários fatores, esta vibração pode ser irritante para aqueles que estão ocupando a área.

Apesar da inquestionável importância do tema, tanto a NBR 6118, quanto a NBR 8800 são bastante superficiais no tratamento do conforto humano, não dando a ele o destaque merecido. Devido a essas limitações, decidimos dar maior ênfase neste trabalho a normais internacionais, as quais possuem uma abrangência maior do que os critérios empregados no Brasil. Portanto, os resultados encontrados neste trabalho serão comparados com os valores limites fornecidos por normas e guias de projetos internacionais (Bachmannet al.(1987), AISC/CISC Design Guide 11 (MURRAY et. al2003), SCI Publication P354 (Smith et al(2009)) e BS 6472 (1992)) e serão expressos em função de uma porcentagem da aceleração da gravidade $(\% \mathrm{~g})$.

\section{METODOLOGIA}

Neste trabalho foi utilizado o modelo proposto por Varela (2004). Nele é considerada a variação da posição da carga com o tempo, permitindo uma representação mais realista da caminhada. Na Figura 4 a carga é constantemente aplicada à estrutura a certa velocidade. Esta carga "P" é representada pela Equação 2.

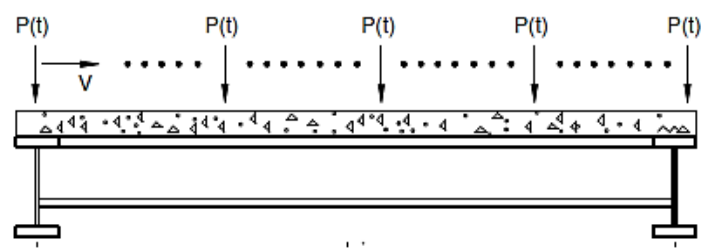

FIGURA 4: Representação da' carga durante a caminhada. FONTE: (Mello, 2009).
$\mathrm{Na}$ análise numérica foi simulada uma caminhada com frequência de $2,26 \mathrm{~Hz}$, ou seja, um período de $1 / f=0,4425 \mathrm{~s}$. Interpolando os valores na Tabela 2, encontrou-se $\mathrm{V}=2,10 \mathrm{~m} / \mathrm{s}$ e $\mathrm{d}=0,966 \mathrm{~m}$. Adotou-se a seguinte simplificação: 0 passo foi dividido em cinco espaços de 0,966/(5=0,193 m), sendo que, a cada ponto foi associada uma carga. Teremos então que cada carga (P1, P2, P3, P4 e P5) deverá ser aplicada durante $0,4425 / 5=0,08850 \mathrm{~s}$, conforme mostrado Figura 5. Entretanto, as cargas não serão executadas simultaneamente. A primeira seria a P1, por 0,0885 s. Ao final desse tempo ela passa a ter o valor de 0,0 e a carga P2 entra em ação por $0,0885 \mathrm{~s}$. Assim, sucessivamente, todas serão aplicadas, varrendo a estrutura de um lado a outro.

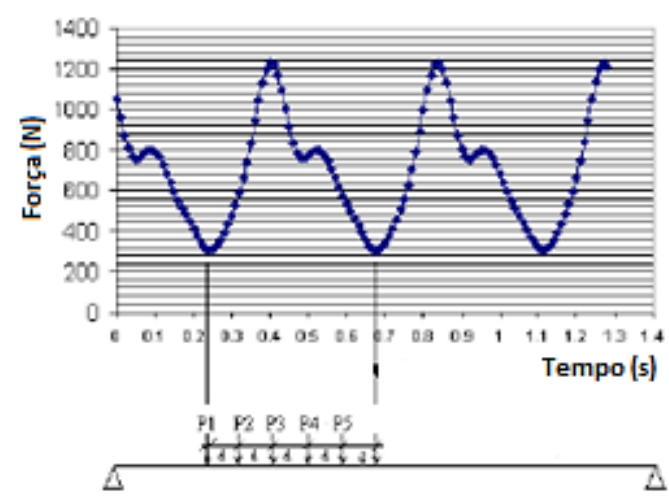

FIGURA 5:Aplicação da carga variando a posição. FONTE: (MOREIRA, 2004).

A primeira força (P1) foi definida com equivalente ao máximo valor da reação do piso, o qual ocorre em $\mathrm{t}=0,0177 \mathrm{~s}$. Para encontrar os demais tempos, foram feitos incrementos de $0,08850 \mathrm{~s}$.

\subsection{MODELO COMPUTACIONAL}

Para a realização da análise dinâmica, utilizou-se técnicas modernas de modelagem, por meio do Método de Elementos Finitos (MEF). Foi usado o SAP2000, pacote de software da Computer and Strutures Inc., para análise estrutural.

No presente trabalho, as vigas e pilares foram simulados com elementos tipo Frame, já nas lajes foram utilizados elementos tipo Shell com comportamento de casca, membrana ou placas, permitindo assim adotar ações normais ao plano. 0 Shell tipo casca foi montado a partir de três ou 
quatro nós, que combinam separadamente os comportamentos de membrana e de flexão das placas.

\subsection{ANÁLISE DINÂMICA DA ESTRUTURA}

O Fanella e Mota (2015) relatam em sua obra que numerosos recursos e métodos estão disponíveis para determinar a frequência de um sistema, mas que geralmente é conveniente obter esta e outras características de vibração a partir de um programa de computador comercial. Dessa forma, neste artigo a resposta dinâmica da passarela foi encontrada mediante uma análise numérica, por meio do método dos elementos finitos, utilizando para tal o programa SAP 2000. Com o objetivo de se avaliar os níveis de vibração do sistema estrutural em estudo, obteve-se inicialmente a resposta dinâmica do mesmo, com base na análise das frequências naturais, deslocamentos, velocidades e acelerações máximas (acelerações de pico). Segundo Ahmed e Badaruzzaman (2011), A frequência natural é aquela que a estrutura vibrará quando deslocada e liberada rapidamente. As estruturas, embora possuam muitas frequências naturais; a mais baixa ou a Frequência "fundamental" é a que merece maior atenção na análise dinâmica, pois, por possuir menor energia, tornam-se mais fácil de ser excitada.

Neste trabalho as acelerações verticais máximas da passarela serão calculadas e comparadas com os limites propostos por normas e recomendações de projeto (Bachmann et al.(1987), AISC/CISC Design Guide 11 (MURRAY et. Al 2003), SCl Publication P354 (Smith et al(2009)) e BS 6472 (1992)).Tal comparação é feita objetivando verificar a ocorrência de níveis de vibrações indesejáveis e desconforto humano.

\section{ESTUDO DE CASO DE UMA PASSARELA EM ESTRUTURA METÁLICA}

\subsection{DESCRIÇÃO DA ESTRUTURA}

O sistema estrutural em estudo se baseia em uma passarela construída em estrutura metálica, com pilares em concreto. A estrutura estudada apresenta 1,2 $\mathrm{m}$ de largura e 26,5 m de comprimento, tendo sido construída com vigas laminadas e pilares em concreto armado. As vigas longitudinais, da referida construção, são perfis W310x21, já as transversais são W150x13. A Figura 6 apresenta uma planta baixa do projeto da estrutura, já a Figura 7 indica o detalhamento do pilar de concreto.

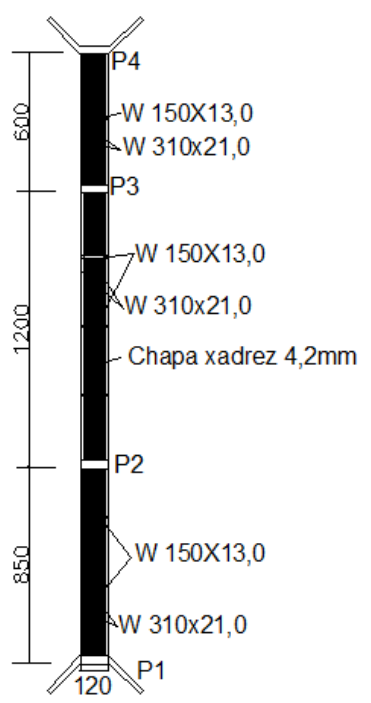

FIGURA 6: Planta baixa da passarela FONTE: Autoria Própria (2017).

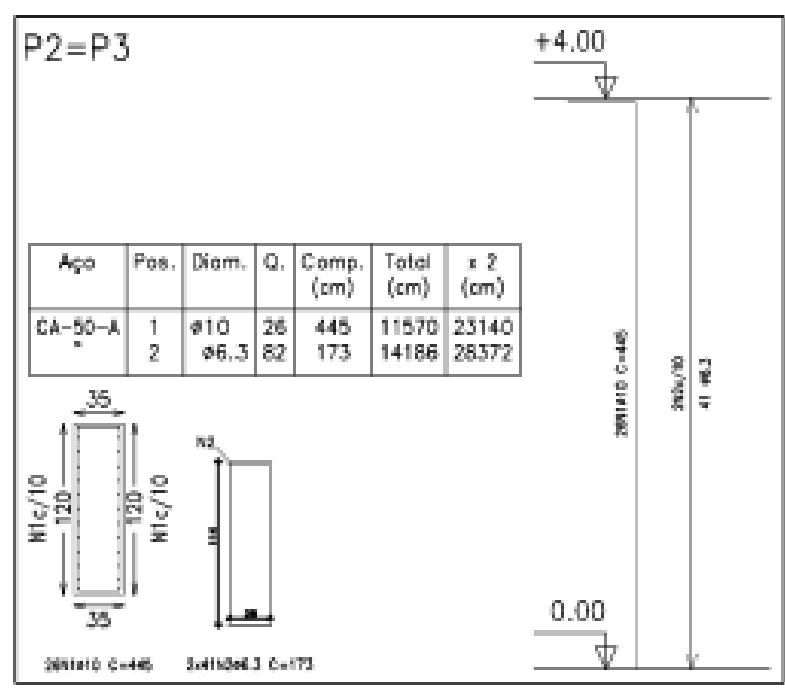

FIGURA 7: Planta de forma dos pilares P2 e P3, em concreto armado. FONTE: Autoria Própria (2017)

Com referência as características físicas dos materiais utilizados, o concreto possui resistência característica à compressão igual a 30,0 MPa e módulo de elasticidade de 31,320 GPa. Os perfis metálicos do tipo "I" são constituídos pelo aço A572Gr50, com limite de escoamento de $345 \mathrm{MPa}$. 
Para as vigas foi considerado um módulo de elasticidade de 200.000,0 MPa.

\subsection{MODELO COMPUTACIONAL}

Para a realização da análise dinâmica, utilizou-se técnicas modernas de modelagem, por meio do Método de Elementos Finitos (MEF). Foi usado o SAP2000, pacote de software da Computer and Strutures Inc., para análise estrutural.

No presente trabalho, as vigas e pilares foram simulados com elementos tipo Frame, já nas lajes foram utilizados elementos tipo Shell, com comportamento de casca, membrana ou placas, permitindo assim adotar ações normais ao plano. $\mathrm{O}$ Shell tipo casca foi montado a partir de três ou quatro nós, que combinam separadamente os comportamentos de membrana e de flexão das placas. Na modelagem desta estrutura não foi considerado o corrimão da passarela na análise de vibrações, sendo que uma eventual modelagem deste elemento poderia contribuir para elevação da rigidez da estrutura.

\subsection{ANÁLISE DINÂMICA DA ESTRUTURA}

O Fanella e Mota (2015) relatam em sua obra que numerosos recursos e métodos estão disponíveis para determinar a frequência de um sistema, mas que geralmente é conveniente obter esta e outras características de vibração a partir de um programa de computador comercial. Dessa forma, neste artigo a resposta dinâmica da passarela foi encontrada mediante uma análise numérica, por meio do método dos elementos finitos, utilizando para tal o programa SAP 2000. Com o objetivo de se avaliar os níveis de vibração do sistema estrutural em estudo, obteve-se inicialmente a resposta dinâmica do mesmo, com base na análise das frequências naturais, deslocamentos, velocidades e acelerações máximas (acelerações de pico). Segundo Ahmed e Badaruzzaman (2011), A frequência natural é aquela que a estrutura vibrará quando deslocada e liberada rapidamente. As estruturas, embora possuam muitas frequências naturais; a mais baixa ou a Frequência "fundamental" é a que merece maior atenção na análise dinâmica, pois, por possuir menor energia, tornam-se mais fácil de ser excitada.

Neste trabalho as acelerações verticais máximas da passarela serão calculadas e comparadas com os limites propostos por normas e recomendações de projeto (Bachmann et al.(1987), AISC/CISC Design Guide 11 (MURRAY et. al2003), SCI Publication P354 (Smith et al(2009)) e BS 6472 (1992)).Tal comparação é feita objetivando verificar a ocorrência de níveis de vibrações indesejáveis e desconforto humano.

\subsection{ANÁLISE DAS FREQUÊNCIAS NATURAIS E DOS MODOS DE VIBRAÇÃO}

As simulações numéricas realizadas ao longo do estudo permitiram a definição das frequências naturais da passarela, como pode ser visto na Tabela 3. As Figuras 8 a 11 indicam os modos de vibração correspondentes às seis primeiras frequências naturais do modelo estrutural.

TABELA 3: Frequências naturais de vibração para os Modos de vibração (Hz).

Frequências Naturais

Modo de vibração

\begin{tabular}{lcc}
\hline$f_{01}$ & 8,25 \\
$f_{02}$ & 9,60 \\
$f_{03}$ & 16,97 \\
$f_{04}$ & 19,30 \\
$f_{05}$ & 21,72 \\
$f_{06}$ & 22,23 \\
\hline
\end{tabular}

\section{Frequência}

FONTE: Autoria Própria (2017). 
Na Figura 8 há predominância de flexão no vão central, que coincidentemente também é o maior. Esse comportamento já era esperado, pois é esse também o trecho com menor rigidez. No segundo modo de vibração, Figura 10, há também predominância de flexão, mas no primeiro vão, isso também já era previsível, posto que esse seja o segundo maior vão. No terceiro modo, Figura 11, ocorre torção no vão central da passarela. No quarto modo, Figura 12, ocorre novamente flexão, mas agora no terceiro vão. No quinto modo, Figura 13, há torção no primeiro vão da passarela. Finalmente, na Figura 14 percebemos a predominância de flexão no vão central, com a formação de dois harmônicos.

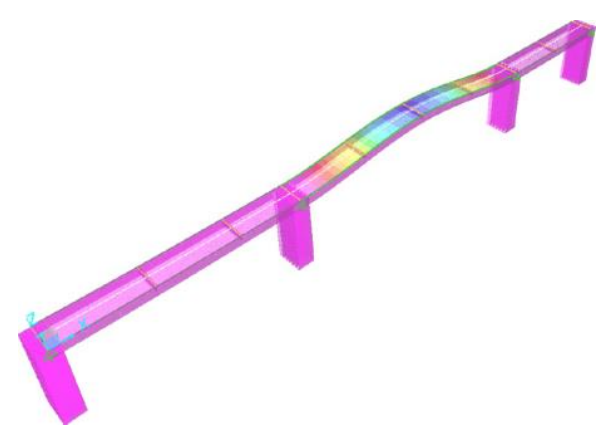

FIGURA 8:Primeiro modo de vibração, frequência 8,25 $\mathrm{Hz}$.

FONTE: Autoria Própria (2017).

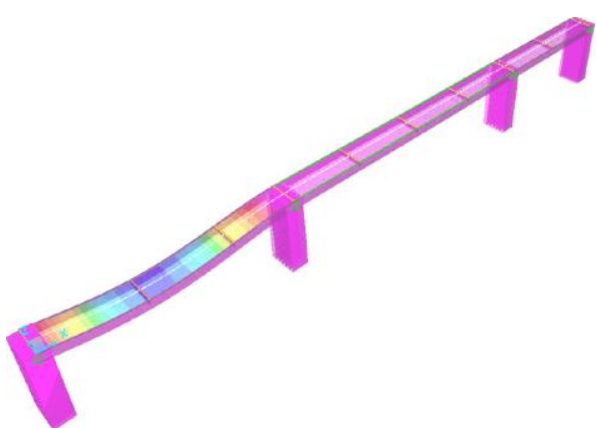

FIGURA 9:Segundo modo de vibração, frequência 9,60 $\mathrm{Hz}$.

FONTE: Autoria Própria (2017).

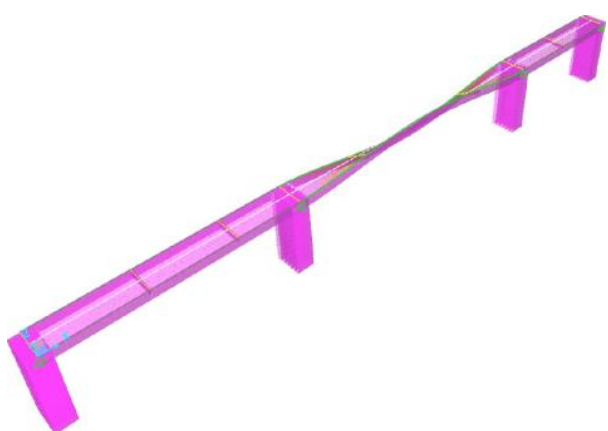

FIGURA 10:Terceiro modo de vibração, frequência 16,97 $\mathrm{Hz}$.

FONTE: Autoria Própria (2017).

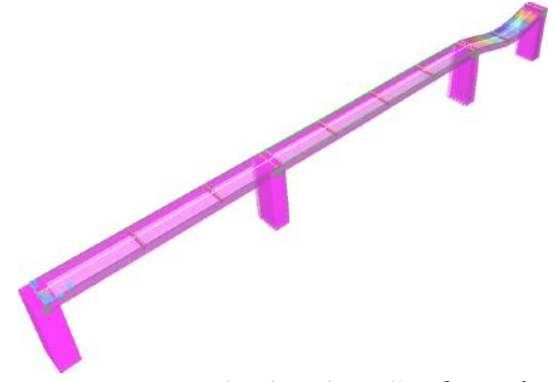

FIGURA 11: Quarto modo de vibração, frequência 19,30 $\mathrm{Hz}$.

FONTE: Autoria Própria (2017).

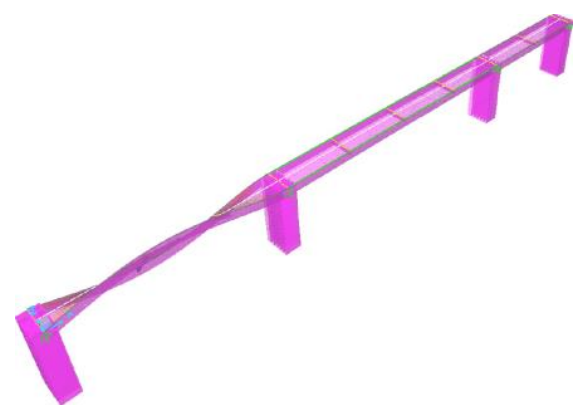

FIGURA 12:Quinto modo de vibração, frequência 21,72 $\mathrm{Hz}$.

FONTE: Autoria Própria (2017).

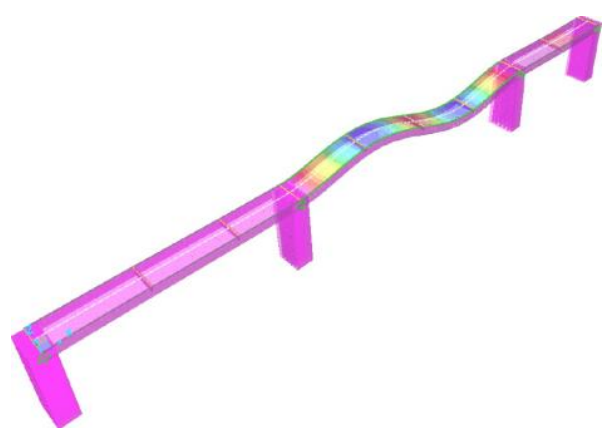

FIGURA 13:Sexto modo de vibração, frequência 22,23 $\mathrm{Hz}$.

FONTE: Autoria Própria (2017)

A análise dos modos de vibração é bastante relevante em um projeto estrutural, já que por meio dela é possível definir os melhores locais para se reformar a estrutura e assim aumentar sua frequência natural. Após exame das imagens, podese inferir que um dos pontos críticos da passarela é o vão central, portanto, um reforço nesse local provavelmente implicaria numa melhoria do comportamento dinâmico.

\subsection{ANÁLISES DAS ACELERAÇÕES MÁXIMAS}

Foram obtidas numericamente as acelerações máximas do sistema, as quais foram comparadas com os valores limites propostos por 
Murray et al (1997). De acordo com Ahmed e Badaruzzaman (2011), O amortecimento da estrutura é importante na atenuação da sua resposta excessiva às vibrações. Experimentalmente ele geralmente é determinado a partir do decaimento da vibração após um impacto. De acordo com Gandomkar et al. (2012) os índices de amortecimento são geralmente medidos a partir da experiência ou adotados por meio de valores sugeridos por guias de projeto e não podem ser determinados através de métodos analíticos. $\mathrm{Na}$ presente análise foi considerado um coeficiente de amortecimento para o sistema estrutural de 3,0\%, esse valor foi estabelecido levando em consideração recomendações presentes em Murray (2000). Foi também considerado a travessia de uma pessoa com o peso de $800 \mathrm{~N}$, cruzando a passarela, de um lado ao outro.

As Figuras 15 a 17 ilustram a resposta dinâmica da passarela investigada, em termos de acelerações, ao longo do tempo. Segundo Feldmann et al. (2009), a localização do ponto de analise da excitação estacionária é escolhido onde a maior das amplitudes de vibração é esperada (para pisos regulares é geralmente o meio do piso). Seguindo, portanto, essa recomendação, optou-se por analisar os pontos localizados na região central de cada um dos vãos, Figura 14.

A Tabela 4 apresenta os resultados das acelerações (r.m.s.) e a porcentagem da aceleração da gravidade (\% g), encontrados com subsídio da análise numérica no SAP2000.

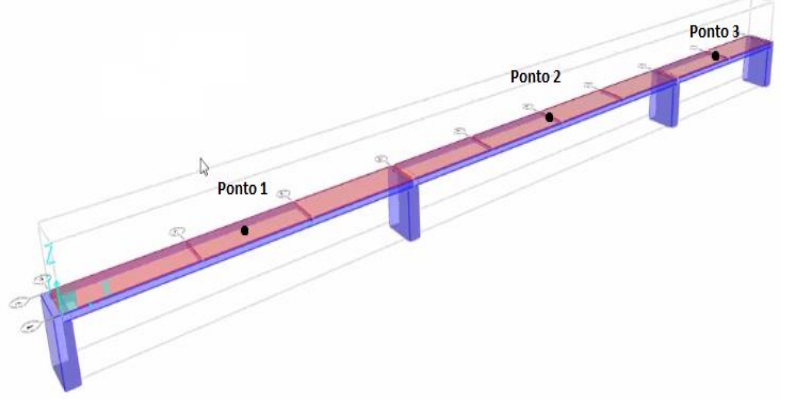

FIGURA 14: Representação esquemática dos Pontos 1, 2 e 3 em que foram medidas os valores da aceleração. FONTE: Autoria Própria (2017)

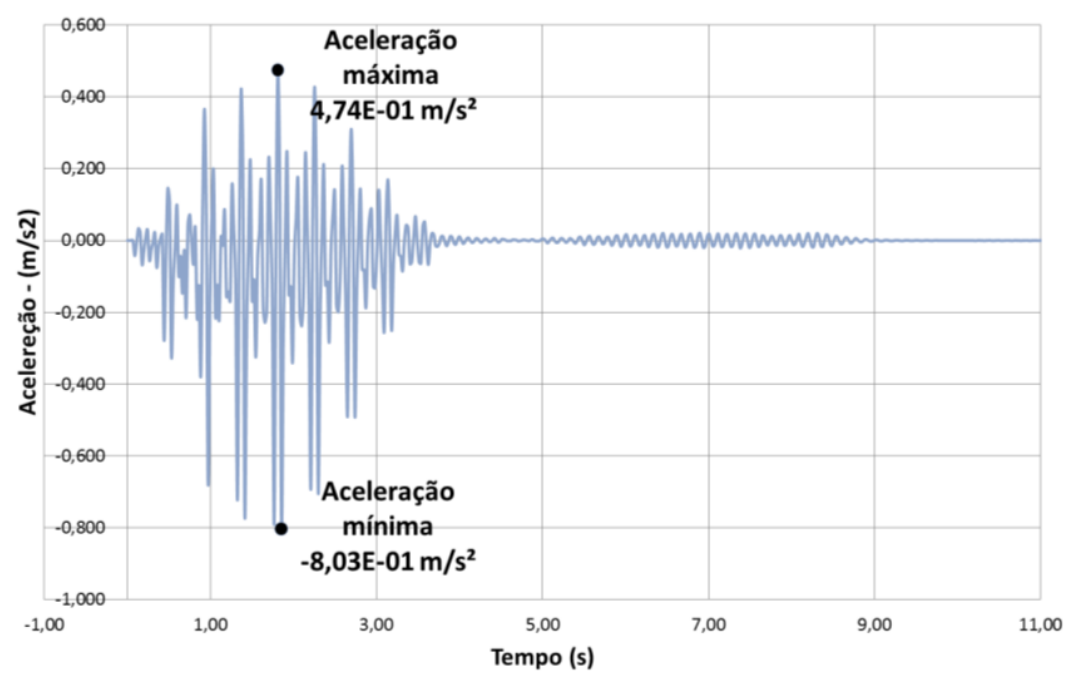

FIGURA 15: Aceleração (r.m.s.) do ponto P1 na direção de $Z$

FONTE: Autoria Própria (2017) 


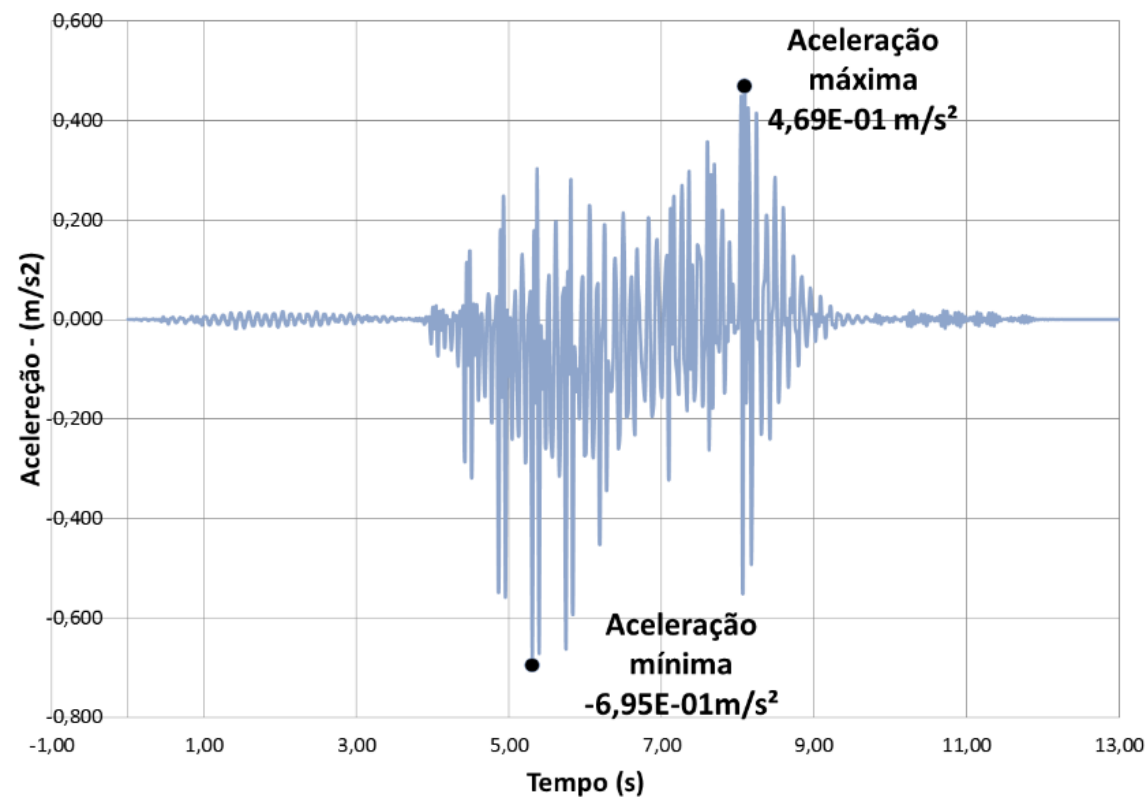

FIGURA 16: Aceleração (r.m.s.) do ponto P2 na direção de Z.

FONTE: Autoria Própria (2017)

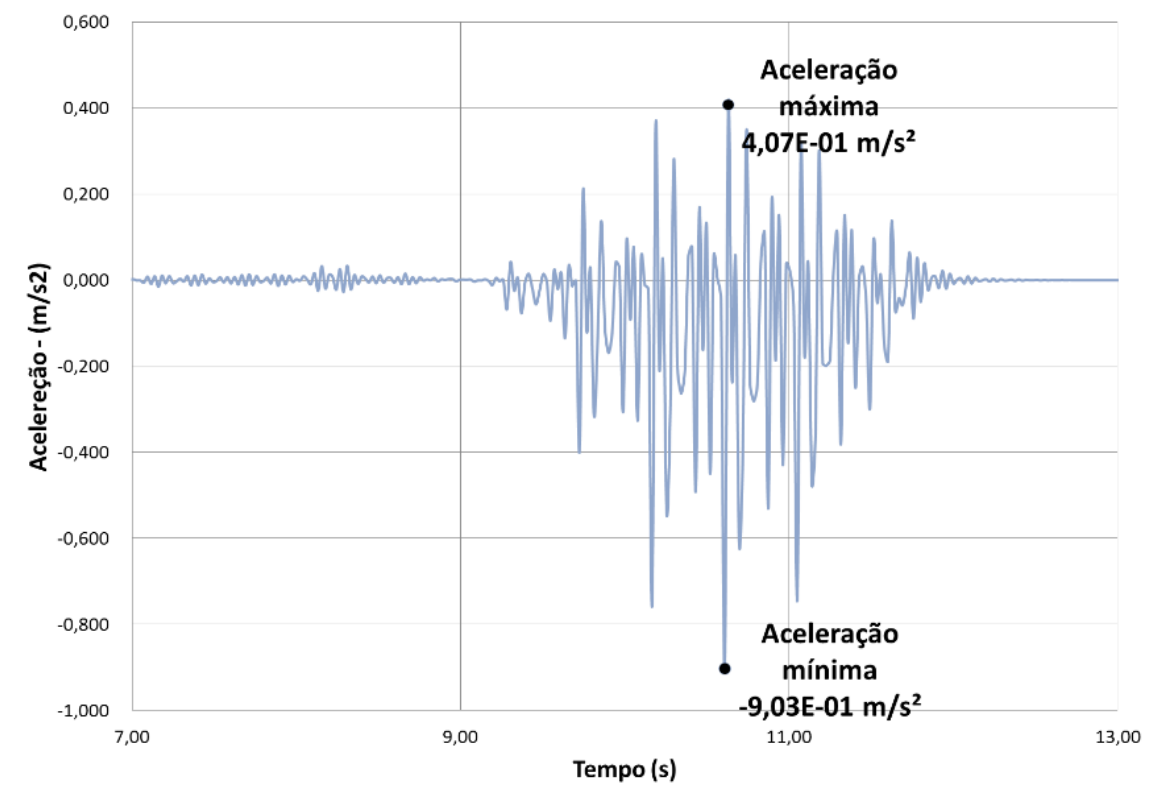

FIGURA 17:Aceleração (r.m.s.) do ponto P3 na direção de Z.

FONTE: Autoria Própria (2017)

Aceleração em r.m.s.

$\left(\mathrm{m} / \mathrm{s}^{2}\right)$

$(\% g)$
P1

0,803

8,03
P2

P3

0,695

0,903

6,95

9,03 
O espectro das respostas dinâmicas da estrutura, em termos das acelerações via análise no domínio da frequência, é apresentado nas Figuras 18,19 e 20.

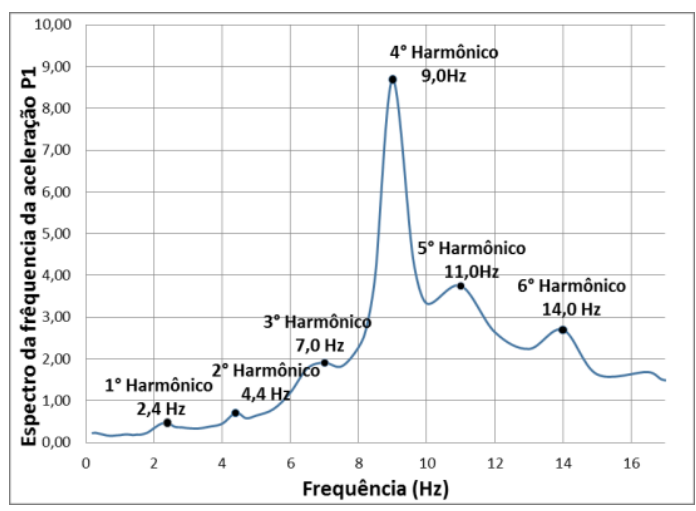

FIGURA 18:Espectro da resposta da aceleração no domínio da frequência para o P1.

FONTE: Autoria Própria (2017).

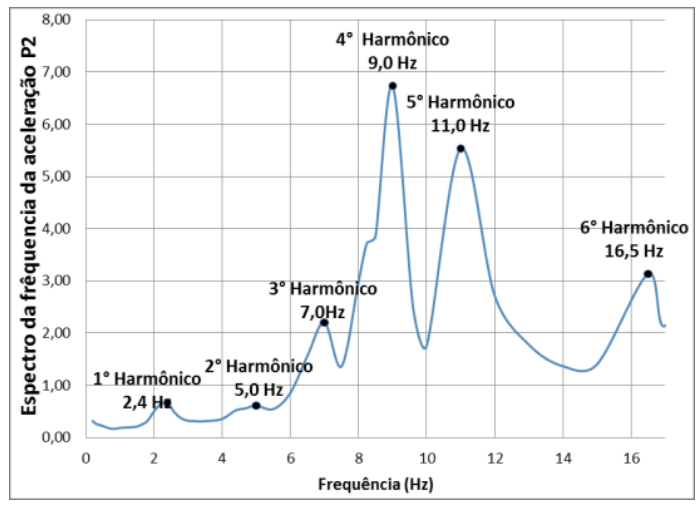

FIGURA 19:Espectro da resposta da aceleração no domínio da frequência para o P2. FONTE: Autoria Própria (2017).

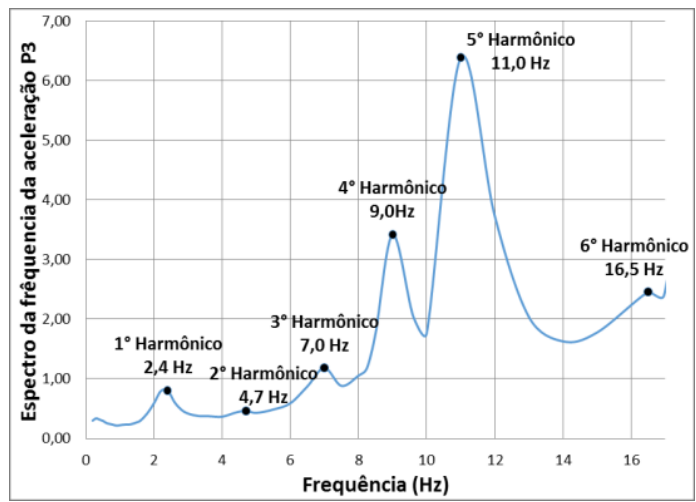

FIGURA 20:Espectro da resposta da aceleração no domínio da frequência para o P3. FONTE: Autoria Própria (2017).

Nas Figuras 18, 19 e 20, apresentam-se o espectro de resposta no domínio da frequência para os pontos $\mathrm{P} 1, \mathrm{P} 2$ e $\mathrm{P} 3$ respectivamente. Pode-se observar nas duas primeiras imagens, que o quarto pico da figura corresponde ao quarto harmônico da excitação produzida pelo caminhar humano, cujo valor é muito próximo à frequência fundamental do sistema de piso ( $f 1=8,25 \mathrm{~Hz}$ ), indicando que este é o maior responsável pela transferência de energia ao sistema. Já no terceiro ponto, é o quinto harmônico da excitação produzida pelo caminhar humano, que é o maior responsável pela transferência de energia ao sistema.

\subsection{ANÁLISE DO CONFORTO HUMANO}

Na Figura 21 é apresentado o resultado da aceleração (r.m.s.) para os pontos P1, P2 e P3, obtidos mediante análise numérica, e suas posições em relação às curvas limites de aceleração, considerando o conforto humano. Esses limites foram definidos, de acordo com normas e guias de projeto internacionais (BACHMANN et al.(1987), ISO/DIS 10137 (2007), AISC/CISC Design Guide 11 (Murray et. al(2003)), SCI Publication P354 (SMITH et al.2009) e BS 6472 1992). A definição dos P1, P2 e P3 e indicação de sua localização na passarela é apresentada na Figura 14.

Pode-se observar na Figura 21 que as acelerações em r.m.s.(\% g) obtidas para todos os pontos estão acima dos limites definidos pelas normas (BACHMANN et al.(1987), AISC/CISC Design Guide 11 (Murray et. al(2003)), SCI Publication P354 (SMITH et al.2009), e BS 6472 1992).Portanto, os resultados obtidos indicam que a passarela em analise viola os critérios de conforto humano, quando submetida a uma carga dinâmica induzida pelo caminhar humano, já que as acelerações produzidas na rampa de acesso analisada estão dentro de uma faixa de vibrações indesejáveis aos seres humanos.

Os resultados desta análise corroboram a necessidade de se considerar a ação dinâmica humana no dimensionamento estrutural. Esse fato fica ainda mais nítido em passarelas de pedestres, construídas em estrutura metálica, as quais por serem cada vez mais esbeltas e flexíveis, são também mais vulneráveis a ações exteriores e seus efeitos dinâmicos. 


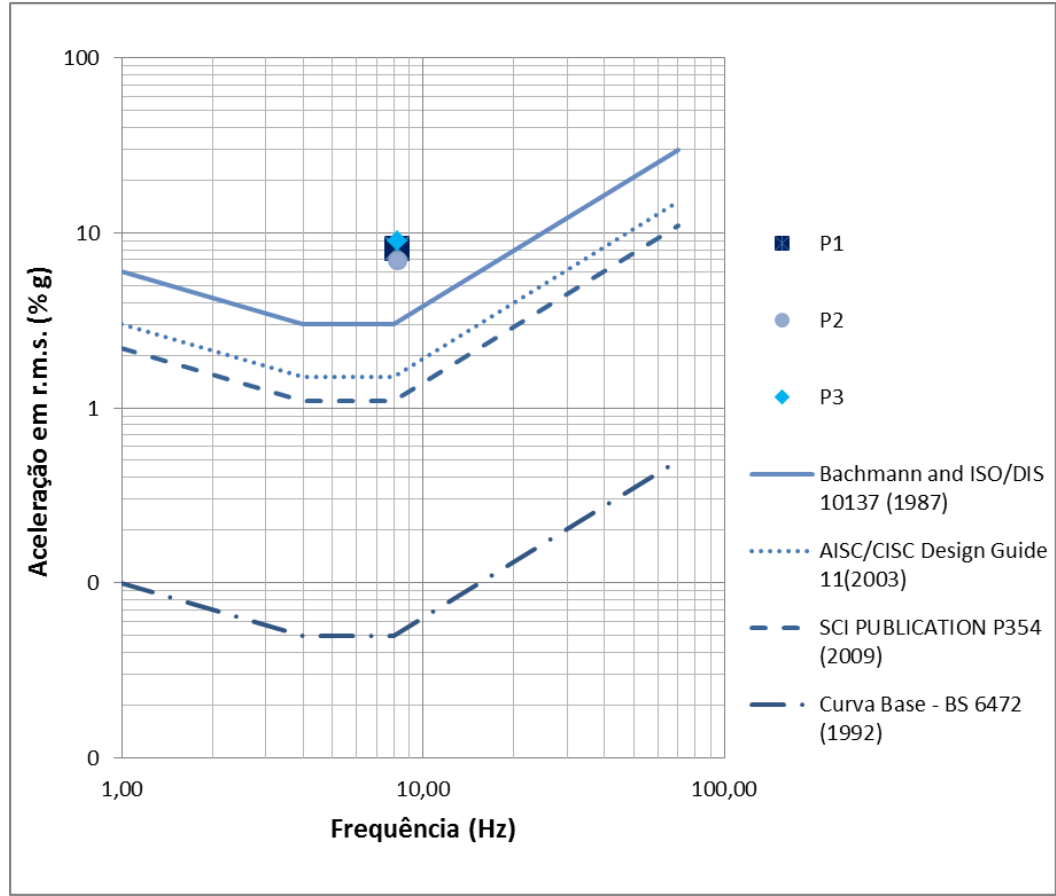

FIGURA 21: Resultado de aceleração (r.m.s.), obtido por meio da análise numérica, representado sobre curvas limites de aceleração, considerando o conforto humano.

\section{CONSIDERAÇÕES FINAIS}

Neste trabalho foi avalia o comportamento estrutural de passarelas de pedestres submetidas ao caminhar humano. A metodologia de analise considera o estudo do comportamento dinâmico de uma passarela mista, submetida a um carregamento dinâmico gerado pela ação dinâmica do caminhar humano.

Utilizando como referenciais recomendações de normas e guias de projeto, nacionais e internacionais, foi criado um modelo computacional, com auxílio do programa computacional SAP2000.Este modelo estrutural baseia-se um uma passarela mista (aço-concreto), com 26,50 m de comprimento e $1,20 \mathrm{~m}$ de largura, a qual possui três vãos contínuos.

Os resultados obtidos por meio dos modelos numéricos foram comparados com os limites propostos por diversas normas e recomendações de projeto internacionais (BACHMANN et al.(1987), ISO/DIS 10137 (2007), AISC/CISC Design Guide 11 (Murray et. al(2003)), SCI Publication P354 (SMITH et al.2009) e BS 6472 1992).Foi constatado, claramente, que a passarela viola os limites de conforto humano, uma vez que apresenta acelerações bem acima dos limites especificados quando submetido ao caminhar de pedestres. É necessário, no entanto reforçar, que a interferência do corrimão da passarela não foi considerada na análise de vibrações, já que este elemento não foi modelado. Uma eventual consideração deste elemento poderia contribuir para elevação da rigidez da estrutura e contribuir para uma possível melhora no comportamento dinâmico da estrutura.

As conclusões obtidas neste artigo reforçam a necessidade de uma análise dinâmica de estruturas mistas de aço-concreto. A consideração apropriada das ações dinâmicas pelos projetistas e, bem como, a correta avaliação da resposta dinâmica deste tipo de estrutura poderiam evitar problemas relacionados ao conforto humano, como os verificados na passarela em estudo.

\section{REFERÊNCIAS BIBLIOGRÁFICAS}

AHMED, Ehsan; BADARUZZAMAN, W.h. Wan. EVALUATION OF NATURAL FREQUENCY AND DAMPING OF PROFILED STEEL SHEET DRY BOARD COMPOSITE PANEL. Journal Of Engineering Science And Technology. Selangor, Malásia, p. 695-708. jun. 2011. Disponível em: <http://jestec.taylors.edu.my/Vol 6lssue 6 December 11/Vol_6_6_695_708_EHSAN AHMED.pdf>. Acesso em: 04 jan. 2017. 
ANUNCIACÃO PINTO, J. F. Análise numérica de vibrações em sistemas de piso misto de aço e concreto e avaliação de desempenho considerando o conforto humano. Dissertação de Mestrado. Pós Graduação em Engenharia Civil, Departamento de Engenharia Civil, Universidade Federal de Viçosa, UFV. Viçosa, MG, Brasil. 2015.

ASSOCIAÇÃO BRASILEIRA DE NORMAS TÉCNICAS. NBR 6118 - Projeto De Estruturas De Concreto. Rio de Janeiro. 2014.

ASSOCIAÇÃO BRASILEIRA DE NORMAS TÉCNICAS. NBR 6118 - Projeto De Estruturas De Concreto. Rio de Janeiro. 2014.

BACHMANN, H.; AMMANN, W. Vibrations in structures induced by man and machines. IABSE Structural Engineering Documents 3edt. Switzerland. 1987.

BRITISH STANDARDS INSTITUTION. BS 6472: Guide to evaluation of human exposure to vibration in buildings (1 Hz to $80 \mathrm{~Hz}$ ) . 1992.

DALLARD, Philippe et al. THE MILLENNIUM BRIDGE, LONDON: PROBLEMS AND SOLUTIONS. The Structural Engineer, London, v. 79, n. 8, p.7-15, 17 abr. 2001. Mensal.

FANELLA, David A.; MOTA, Mike. Vibration of Reinforced Concrete Floor Systems. Structure Magazine, Algonguin, v. 8, n. 8, p.15-16, abr. 2015. Disponível em: <http://www.structuremag.org/?p=8402>. Acesso em: 04 jan. 2017.

FIGUEIREDO, Fábio Pereira. Estudo do comportamento dinâmico de passarelas devido ao caminhar de pedestres. 2005. 133 f. Dissertação (Mestrado) - Curso de Engenharia Civil, Centro de Tecnologia e Ciências, Universidade do Estado do Rio de Janeiro, Rio de Janeiro, 2005.

FELDMANN, M. et al. Design offloorstructures for humaninducedvibrations: Background document in supporttotheimplementation,

harmonizationandfurtherdevelopmentoftheEurocodes.

Luxembourg: Jrc - Eccs, 2009. 75 p. Disponível em: $<$ http://eurocodes.jrc.ec.europa.eu/show_Entity.php?fil e_id=EC_00000069.>. Acesso em: 04 jan. 2017.

FULLER, Robert G. et al. TACOMA NARROWS BRIDGE COLLAPSE. Lincoln: Aapt-american Association Of Physics Teachers, 1994.27 p. Disponível em: <https://www.aapt.org/Store/upload/tacoma_narrows2 .pdf>. Acesso em: 09 abr. 2017.

GANDOMKAR, Farhad Abbas et al. Dynamic response of low frequency Profiled Steel Sheet Dry Board with Concrete infill (PSSDBC) floor system under human walking load. Latin American Journal Of Solids And Structures: On-line version ISSN 1679-7825, Rio de Janeiro, v. 9, n. 1, p.21-41, 3 jan. 2012. Disponível em: <http://www.scielo.br/scielo.php?script=sci_arttext\&pid
=S1679-78252012000100002>. Acesso em: 04 jan. 2017.

MANGERIG, Ingbert; ZAPFE, Cedrik. Vibration and Damping of Steel Constructions. In: THE GERMANJAPANESE BRIDGE SYMPOSIUM, 7., 2007, Osaka, Japan. Anais... . Osaka: Osaka City University, Japan, 2007. p. 10 - 18. Disponível em: <http://www.ingzapfe.com/pdf/publikationen/9_Vibration_and_dampin g_of_steel_constructions.pdf>. Acesso em: 04 jan. 2017.

MELLO, A. V. A. Vibrações em Pisos de Edificações Induzidas por Atividades Humanas. Dissertação de Mestrado. Faculdade de Engenharia, Programa de PósGraduação em Engenharia Civil,Universidade Estadual do Rio de Janeiro, UERJ. Rio de Janeiro, RJ, Brasil. 2005.

MELLO, A. V. A. Análise do Efeito da Interação AçoConcreto sobre a Resposta Dinâmica de Pisos Mistos. Tese de Doutorado. Programa de Pós-Graduação em Engenharia Civil, PUC-Rio. Rio de Janeiro, RJ, Brasil. 2009.

MOREIRA, B. C. Avaliação Comparativa de Pisos de Edificações em Estrutura Metálica Quanto aos Critérios de Vibração. Dissertação de Mestrado. Departamento de Engenharia Civil, UFOP. Ouro Preto, MG, Brasil. 2004.

MURRAY, T. M.; ALLEN, D. E.; UNGAR, E. E. Floor vibrations due to human activity. Chicago, USA, Steel design guide series $\mathrm{n}$ - 11, American Institute of Steel Construction, AISC. 2003

Murray, T.M. (2000). Floor vibrations: 10 Tips for designers of office buildings. Structure/ fall 2000, 26-30.

SAP2000. Nonlinear Version 15.2. User's Manual, version 15.2. Inc., Berkeley, CA. 2012.

SMITH, A. L.; HICKS, S. J.; DEVINE, P. J. Design of floors for vibration: A new approach; Volume 354. The SCI publication. p 114. Steel Construction Institute. Silwood Park, Ascot, Berkshire. 2009.

VARELA, W. D. Modelo Teórico-Experimental para Análises de Vibrações Induzidas por Pessoas Caminhando Sobre Lajes De Edifícios, 2004. 309f. Tese de Doutorado da Coppe/UFRJ, Rio deJaneiro, Rj, Brasil, 2004. 